\title{
De Molinari a Ravignani: el caso del Instituto de Historia Argentina y Americana (1974-1977)
}

Molinari to Ravignani's: the case of the Institute of Argentine and American History (1974-1977)

\author{
María Elena García Moral \\ Programa de Investigaciones en Historiografía Argentina-Universidad Nacional de Buenos Aires- \\ Instituto de Historia Argentina y Americana "Dr. Emilio Ravignani"-CONICET, Argentina \\ maegm@yahoo.com.ar
}

\section{ReSUMEN:}

Se estudia la situación del Instituto de Historia Argentina y Americana de la FFyL de la UBA en el período 1974-1977, es decir, tras la intervención de Alberto Ottalagano y hasta la muerte de quien fue su director por más de veinte años, Ricardo Caillet Bois. Se han utilizado preferentemente los materiales disponibles en los Archivos del Instituto Ravignani y de la FFyL. Se trató de un momento de revisión y reacción, durante el cual el Instituto conoció continuidades y cambios en su dirección, personal, investigaciones, publicaciones y actividades, como el de su propia nomenclatura: de Molinari a Ravignani. Palabras claves: Instituto de Historia, Historiografía, Argentina, Ibarguren, Caillet Bois.

Palabras ClaVE: Instituto de Historia, Historiografía, Argentina, Ibarguren, Caillet Bois.

\section{ABStract:}

Examines the situation of the Institute of Argentine and American History of the FFyL of the UBA in 1974-1977 period, that is, after the intervention of Alberto Ottalagano and even death for whom he was director for over twenty years, Ricardo Caillet Bois. Materials available in the files of the Ravignani Institute and the FFyL have been preferably used. It was a time of review and reaction, during which the Institute met continuities and changes in their direction, personal, researchs, publications and activities, as its own nomenclature: Molinari to Ravignani's.

KEYWORDS: Institute of History, Historiography, Argentina, Ibarguren, Caillet Bois.

\section{INTRODUCCIÓN}

El actual Instituto de Historia Argentina y Americana "Dr. Emilio Ravignani”, creado en 1921 por las autoridades de la Facultad de Filosofía y Letras (FFyL) de la Universidad de Buenos Aires (UBA), estuvo bajo la dirección de Emilio Ravignani -uno de los miembros de la llamada Nueva Escuela Histórica (NEH), de filiación radical- hasta 1946. Durante los años del primer peronismo, la dirección del mismo estuvo a cargo brevemente del revisionista Vicente Sierra y luego de Diego Luis Molinari -otro miembro de la NEH de pasado radical, aunque por entonces adscripto al peronismo y cercano al revisionismo histórico-. Tras el derrocamiento de Juan D. Perón en 1955 y hasta 1973, ocupó la dirección del Instituto un discípulo de Ravignani, Ricardo Caillet Bois, y fue entonces que, en homenaje a quien fuera su primer director, el Instituto recibió el nombre de Emilio Ravignani (Buchbinder, 1997 y 2006, p. 113; Devoto, 2006, pp. 12-13; Pagano y Galante, 1993; Devoto y Pagano, 2009).

Entre 1973 y 1974, bajo la experiencia de la llamada Universidad Nacional y Popular de Buenos Aires, no sólo cambió su denominación por la de "Diego Luis Molinari", sino que conoció en muy pocos meses -entre junio de 1973 y mayo de 1974-, primero, la intervención de Eduardo Luis Duhalde -en colaboración con Rodolfo Ortega Peña- y, luego, la co-dirección de Hebe Margarita García y Ana Lía Payró. En septiembre 
de 1974, el gobierno de María Estela Martínez de Perón intervino a la UBA dejando cesantes a todas sus autoridades. En el caso del Instituto, tras una reorganización en la que cumplió algunas funciones Jorge Augusto Ocón, fue nombrado al frente del mismo Federico Ibarguren. Luego del golpe de Estado de marzo de 1976, Caillet Bois retornó a la dirección que ocupó hasta su muerte, acaecida en julio de 1977.

En cierta forma, en otro trabajo hemos revisado la situación del Instituto durante aquella singular coyuntura de 1973-1974. ${ }^{1}$ En esta ocasión, nos proponemos acercarnos a esos momentos de revisión y de reacción que supusieron su reorganización y luego las gestiones de Ibarguren primero y de Caillet Bois después. Para poder estudiar la actuación de estos sectores en el Instituto hemos consultado no sólo resoluciones institucionales y publicaciones periódicas - como el Boletín del mismo Instituto, sino el Archivo institucional (AIR) donde hemos encontrado una serie de materiales inéditos sobre los momentos y las gestiones analizadas, que hacen posible una primera reconstrucción de redes y trayectorias.

\section{EL MARCO POLÍTICO-INSTITUCIONAL}

Tras la muerte de Juan D. Perón y en medio de un proceso de aumento de la represión estatal y paraestatal, en agosto de 1974 el gobierno de "Isabel" Perón decidió reemplazar al ministro de Educación Jorge Alberto Taiana por Oscar Ivanissevich, un representante del nacionalismo católico de derecha. La designación del ministro fue resistida por parte de estudiantes y docentes de la UBA que, entre fines de agosto y principios de septiembre, organizaron clases públicas, movilizaciones y la toma del Rectorado y de varias facultades. Las medidas adoptadas contaron con el apoyo público de algunas autoridades como el mismo rector, Héctor Laguzzi, la decana de la FFyL, Adriana Puiggrós, y el decano de la Facultad de Arquitectura, Alfredo Ibarlucía. No sólo las medidas fueron reprimidas mientras se generalizaban las detenciones y los allanamientos en las facultades, sino que a principios de septiembre se produjeron atentados contra la decana Puiggrós, el rector del Colegio Nacional de Buenos Aires, Raúl Aragón y el propio Laguzzi que sufrió el asesinato de su hijo menor (Friedemann, 2016; Besoky, 2017).

Con todo, el 17 de septiembre mediante un decreto firmado por la presidente y el ministro Ivanissevich se intervino la UBA y se designó como rector interventor hasta el 31 de diciembre de ese mismo año al abogado Alberto Ottalagano, autodenominado fascista y reconocido militante del peronismo de derecha (Friedemann, 2016; Besoky, 2017; Seia, s/f.). En buena medida, sus nombramientos formaron parte de las medidas de depuración que se aplicaron no sólo al interior del movimiento peronista, sino en la administración pública y en las universidades nacionales a través de las intervenciones. Según nos informa Sergio Friedemann (2016), las lecturas tanto de la izquierda como de la derecha del peronismo coincidían en que "las universidades fueron el último bastión que pudo conservar la 'tendencia revolucionaria' del peronismo" (pp. 14-15).

La gestión de Ottalagano se caracterizó por su sesgo nacionalista, clerical y anticomunista. Entre las medidas adoptadas se destacan la imposición de un asueto hasta mediados de octubre en todos los establecimientos dependientes de la UBA, excepto los hospitales; la derogación de la mayoría de las resoluciones aprobadas desde el rectorado de Rodolfo Puiggrós (junio-octubre de 1973), incluidos los nombramientos docentes interinos; y la remoción de todos los decanos y funcionarios jerárquicos, las cesantías masivas y el nombramiento de nuevas autoridades, en general pertenecientes a la derecha peronista como fue el caso del jesuita Raúl Sánchez Abelenda designado al frente de la FFyL.

Justamente, como parte de sus reformas institucionales, Ottalagano dispuso el traspaso de las carreras de Ciencias de la Educación, Psicología y Sociología, consideradas más conflictivas, desde el ámbito de la FFyL a la órbita directa del Rectorado (Friedemann, 2016). Al parecer, también dio directivas para que en los programas de Historia y Geografía de los colegios dependientes de la universidad se destacaran especialmente las acciones bélicas de las fuerzas armadas, así como solicitó al Ministerio de Defensa que designase a tres historiadores y geógrafos militares para formar a los rectores de los institutos (Besoky, 2017). 
Entre tanto, se sucedieron las amenazas y los atentados contra autoridades, docentes y estudiantes, muchos de los cuales optaron por exiliarse, como fueron los casos de Taiana, Laguzzi, Puiggrós -Rodolfo y Adrianae Ibarlucía.

Casi al término de la intervención de Ottalagano, un decreto del Poder Ejecutivo prorrogó la intervención de la UBA por un año más, hasta el 31 de diciembre de 1975, y nombró como rector normalizador a Julio Lyonnet, quien continuó con la misma orientación. Por otra parte, en agosto de 1975 Ivanissevich dejó su cargo al frente del ministerio. En su lugar asumió Pedro J. Arrighi, manteniendo una línea católica de derecha y nombrando a un nuevo rector normalizador de la UBA, Eduardo Mangiante, que fue sucedido por José Alocén hasta el golpe de Estado del 24 de marzo de 1976 (Seia, s/f.).

Tras el golpe de 1976 el Ministerio de Cultura y Educación fue gestionado por cinco ministros civiles y, durante dos períodos breves, por el Ministro del Interior, el general de brigada Albano E. Harguindeguy. El 27 de marzo de 1976 fue designado como delegado militar en la UBA el capitán de navío Edmundo E. Said, quien ocupó ese cargo hasta el 6 de agosto de ese año cuando fue reemplazado por el ingeniero civil Alberto Constantini. Este último renunció al mes de haber asumido el cargo, siendo reemplazado interinamente por el secretario de Ciencia y Tecnología, Sol Rabasa. En marzo de 1977 fue el turno de Luis Carlos Cabral, quien posteriormente fue destituido y también reemplazado interinamente por Alberto V. Donnes, entonces decano de la Facultad de Medicina. ${ }^{2}$ Estos cambios supusieron no sólo tensiones sino también reorientaciones.

¿Cómo se vio afectada la situación del Instituto de Historia Argentina y Americana tras la intervención de Ottalagano, el decanato de Sánchez Abelenda y el golpe de Estado de marzo de 1976? ¿Qué cambios y qué continuidades pueden observarse tanto en su dirección y personal como en sus investigaciones, publicaciones y actividades?

\section{El Instituto Molinari tras la intervención de OtTalagano y durante la gestión DE IBARgUREN}

Como hemos visto, durante la intervención de Ottalagano, en la FFyL fue designado como delegado interventor el presbítero Sánchez Abelenda (AIR 46, Resolución (CS) N 17, 24/9/1974, folio 436). En agosto de 1975, acompañando la designación de Mangiante como rector normalizador, Sánchez Abelenda fue designado decano normalizador, cargo que ocupó hasta el 24 de marzo de 1976.

Durante su gestión el cuadro directivo de la Facultad tuvo relativa estabilidad y las nuevas autoridades gozaron de gran libertad para la designación del cuerpo docente, porque todos los docentes de la UBA habían sido declarados en comisión suspendiendo la estabilidad de sus cargos (Mallimaci y Giorgi, 2007, p. 9).

En diciembre de 1974, Sánchez Abelenda, nombró al antropólogo Marcelo Bórmida como director del Departamento de Ciencias Históricas, ${ }^{3}$ así como solicitó a la UBA dejar sin efecto la resolución que creaba el Instituto de Estudios Argentinos y Latinoamericanos. (Resolución $N^{\circ} 83$ (bis), 27/12/1974). Cabe recordar que la gestión anterior de la Facultad, comandada por la decana Adriana Puiggrós, había emprendido una reestructuración de los institutos y centros de estudio e investigación dependientes de la misma, en cuyo marco se había creado el mencionado Instituto de Estudios Argentinos y Latinoamericanos con una serie de centros dependientes ${ }^{4}$ y que, desde entonces, el viejo Instituto de Historia Argentina y Americana había pasado a constituir uno de ellos como Centro de Estudios de Historia Argentina y Latinoamericana, bajo la dirección de su padre, Rodolfo Puiggrós (AIR 46, Resolución $N^{\circ} 355,23 / 7 / 1974$, folios 451-455, y Resolución (CS) N $\mathrm{N}^{\circ}$ 471, 12/8/1974, folio 442). En la citada Resolución de fines de 1974 se esgrimía que la creación de dicho Instituto había sido producto de la "política comunista" instaurada en la Facultad, así como sus Centros 
respondían a la concepción marxista de la Historia y la Sociología que imperaba en la Universidad y que los mismos servían para la difusión de la ideología comunista que se expandía en todos los ámbitos de la Facultad por tratarse de organismos interdisciplinarios (...) (Resolución $\mathrm{N}^{\circ} 83$ (bis), 27/12/1974).

Un día antes de dejar su cargo, Ottalagano aprobó el Reglamento de funcionamiento académico de la FFyL donde se estableció que el Departamento de Ciencias Históricas comprendería tres secciones: Historia, Geografía y Ciencias Antropológicas (AIR 46, Resolución (CS) N437, 30/12/1974, folios 377-383). De hecho, se restableció el organigrama anterior a 1973 en lo que respecta a la estructura de los centros de investigación, pero se modificó el organigrama de los departamentos docentes e institutos de investigación. Por tal motivo, como hemos visto, entonces desaparecieron de la estructura de la Facultad las carreras de Sociología, Psicología y Ciencias de la Educación -posteriormente reinsertada- y la de Antropología quedó supeditada al Departamento de Historia (Mallimaci y Giorgi, 2007, p. 10). Asimismo, fue restituido el Instituto de Historia Argentina y Americana y se mantuvo el nombre "Diego Luis Molinari", sólo que se antepuso el título de "Dr." sin mayores precisiones formales (Mallimaci y Giorgi, 2007, p. 9). También como parte de la reestructuración académica se aprobaron los nuevos planes y programas de estudios de las carreras de la Facultad, incluida la de Historia (AIR 46, Resolución (CS) No 439, 30/12/1974, folios 340 y 356-361). Entonces el plan de estudios de la carrera de Ciencias Históricas se dividió en tres secciones: Historia, Ciencias Antropológicas y Geografía, contando todas ellas con los ciclos introductorio, básico y de especialización, y la de Ciencias Antropológicas asimismo con un ciclo de orientación, precedidos en todos los casos de un primer año común para todos los ingresantes a la UBA (Resolución $N^{\circ}$ 91, 27/12/1974). Posteriormente, fue modificado de nuevo el organigrama de los departamentos docentes de la Facultad, por lo cual el Departamento de Artes pasó a ser una nueva sección del sobredimensionado Departamento de Ciencias Históricas (Resolución No 109, 7/3/1975). Justamente, aduciendo la necesidad de coordinar las múltiples tareas del Departamento de Ciencias Históricas, que pasó a comprender cuatro secciones, se decidió nombrar como asesor general del mismo al profesor Benjamín García Holgado (Resolución № 725 , 16/7/1975).

Como quiera que sea, el 13 de marzo de 1975, Jorge Augusto Ocón, graduado de la carrera de Historia de la Facultad, aficionado al revisionismo histórico y a cargo de Historia Argentina I, fue designado director de la Sección Historia del Departamento de Ciencias Históricas (Resolución No 121, 13/3/1975). ${ }^{5}$ De hecho, Ernesto La Croce, del Departamento de Estudios de Posgrado, le solicitó a Ocón su colaboración para organizar un coloquio sobre "El revisionismo en la historiografía argentina contemporánea", entre cuyos disertantes se sugerían al propio Ocón, al historiador revisionista Julio Irazusta y al profesor de la casa Antonio Jorge Pérez Amuchástegui (AIR 43, 10/6/1975, folio 290). En realidad, unos días antes, Irazusta había sido designado como investigador asimilado a la categoría de profesor titular en el Departamento de Ciencias Históricas, aduciendo "la relevante actuación en el campo de la investigación del ilustre miembro de la Academia Nacional de la Historia" y sus "valiosos aportes y contribuciones en la problemática histórica" "que han trascendido el ámbito nacional para proyectarse internacionalmente con la seguridad del erudito y la plenitud del maestro" (Resolución No 101,7/3/1975).

Entonces Ocón fue varias veces designado para representar a la Facultad en eventos académicos: junto con Beatriz Spota de Lapieza Elli, Olga M. García D Agostino y Raquel Naso ante el Tercer Congreso de Historia Argentina y Regional a celebrarse en Santa Fe y Rosario (Resolución No 660, 3/7/1975); con la profesora Susana Ratto de Sambucetti ante el Primer Congreso Municipal de Historia de la Ciudad de Buenos Aires, organizado por el Instituto Histórico Municipal y la Junta Central de Estudios Históricos; y junto con el doctor Carlos Eduardo Weiss para representar como delegados al decano ante este último Congreso (Resoluciones $N^{\circ} 7$ (bis) y 9 (bis), 10/9/1975).

Si bien no contamos con una Resolución que nombre a Ocón también al frente del Instituto de Historia Argentina y Americana "Dr. Diego Luis Molinari”, es posible que haya cumplido con algunas funciones relativas a su dirección, por lo menos desde julio del mismo año cuando en un pedido de publicaciones 
institucionales cursado por la Biblioteca y Centro de Información Documental del Estado Mayor Conjunto se saludaba su designación también como director del mismo (AIR 43, 17/7/1975 y 25/7/1975, folios 52 y 232).

Entonces fueron muy frecuentes los pedidos de publicaciones cursados a Ocón en su calidad de director tanto del Instituto Molinari, como el del diario La Nación (AIR 43, 28/7/1975 y 4/8/1975, folios 270 y 277), entre otros; ${ }^{6}$ así como de la Sección Historia del Departamento de Ciencias Históricas, como los de la Comisión de Homenaje y Monumento a los Héroes de "Vuelta de Obligado" (AIR 43, 17/9/1975, folio 53), y/o para las bibliotecas del Museo Etnográfico "Juan B. Ambrosetti” de la FFyL de la UBA (AIR 43, 26/3/1975, folio 224), y del Departamento de Historia del Instituto del Profesorado del Consejo Superior de Educación Católica. ${ }^{7}$

Previamente, otras solicitudes de materiales fueron dirigidas a Paulino Ramón Vázquez (AIR 43, 2/9/1975, folio 69), en tanto jefe de publicaciones del Instituto Molinari, como las de los museos Mitre (AIR $43,16 / 4 / 1975$ y $8 / 4 / 1976$, folios 273 y 275 ) y de Arte Decorativo. ${ }^{8}$

Por su parte, Ángel Castellan, a cargo de la cátedra de Teoría e Historia de la Historiografía en la Facultad y entonces también jefe del área de Historia y Museología del Departamento de Política Social de la Universidad Nacional de Luján, solicitó una serie de colecciones del Instituto Molinari con destino a la biblioteca del Centro de Investigaciones Históricas de aquella última. Su nota fechada el 10 de abril de 1975, en cambio, estaba dirigida a Bórmida en tanto director del Departamento de Ciencias Históricas. ${ }^{9}$

En cualquier caso, en tanto director de la Sección Historia, Ocón también se encargó de resolver la intimidación efectuada al Instituto Molinari por parte del Tribunal de Cuentas de la Nación por un saldo del subsidio otorgado al mismo en 1967 para la impresión del cuarto tomo del Archivo del Doctor Rufino de Elizalde (AIR 107, 7/5/1975, s/f.), así como de gestionar ante el decano Sánchez Abelenda las partidas que permitieran solventar la publicación de dos nuevos tomos del mismo Archivo (AIR 107, 20/11/1975, folio 1), aunque en este último caso sin éxito (AIR 107, 4 y 10/12/1975 y 12/5/1976, folio 5).

Continuando con la reestructuración de los centros e institutos dependientes de la Facultad, el 27 de agosto de 1975 Sánchez Abelenda estableció que el Instituto de Historia Argentina y Americana "Dr. Diego Luis Molinari", junto con otros cinco institutos (el Instituto de Historia del Derecho "Ricardo Levene", el Instituto de Historia Antigua Oriental, el Instituto de Historia Antigua Medieval, el Instituto de Historia Contemporánea y el Instituto de Historia de España) dependiera de la Sección Historia del Departamento de Ciencias Históricas (AIR 46, Resolución N 1141, 27/8/1975, folios 291-292).

Ese mismo día fue designado al frente del mismo el escribano Federico Ibarguren, hijo del también historiador revisionista Carlos Ibarguren Uriburu, quien se había desempeñado como docente en la Facultad de Humanidades de la Universidad de La Plata y era miembro del Instituto de Investigaciones Históricas "Juan Manuel de Rosas" (Devoto y Pagano, 2009). ${ }^{10}$ De hecho, aduciendo también sus "valiosos aportes y contribuciones en la problemática histórica”, Ibarguren revistaba como investigador asimilado a la categoría de profesor titular en el Departamento de Ciencias Históricas desde abril (Resolución No 240, 18/4/1975).

A pedido del rector, Sánchez Abelenda también se dispuso a regular el acceso de los alumnos a las dependencias de la FFyL, estableciendo que los estudiantes debían acreditar su condición de tales para el ingreso mediante la exhibición de la libreta universitaria o un documento sustituto (AIR 46, Resolución $\mathrm{N}^{\circ}$ 228, 10/10/1975, folio 284). Asimismo, en el mes de noviembre prescribió la conmemoración en la Facultad del Combate de la Vuelta de Obligado, designando a Roberto H. Marfany para que hago uso de la palabra en el acto académico (AIR 46, 27/11/1975, folio 272).

En cualquier caso, un tiempo después del golpe de Estado, el 6 de mayo de 1976, Federico Ibarguren presentó su renuncia a la dirección del Instituto Molinari (AIR 46, Resolución No 363, 13/5/1976, folio 216). Previamente, en abril del mismo año, habían sido nombradas directora y secretaria del Departamento 
de Historia, con carácter ad honorem, las historiadoras María del Carmen Carlé y Perla Fuscaldo, respectivamente ( Resolución No 90, 20/4/1976).

\section{La vuelta de Caillet Bois}

El 12 de mayo de 1976 volvió a estar al frente del Instituto, nuevamente renombrado "Dr. Emilio Ravignani”, ${ }^{11}$ su antiguo director, Ricardo Caillet Bois. ${ }^{12}$ Desde el 29 de marzo oficiaba como delegado militar de la Facultad el capitán de corbeta Napoleón Claisse que se dispuso a reglamentar el acceso a todas sus dependencias (AIR 46, Resolución (CS) No 54, 29/3/1976, folio 259). Por su parte, Caillet Bois elevó a Claisse un informe del estado de los materiales y publicaciones encontradas en el Instituto al momento de hacerse cargo del mismo, donde denunciaba sobre todo los faltantes (AIR 44, 14/7/1976, folios 150-151).

En octubre presentó la renuncia el decano de la Facultad, Ángel José Battistessa. Arturo Berenguer Carisomo ofició primero como decano sustituto y en noviembre obtuvo el nombramiento oficial de parte del ministro de Cultura y Educación, Ricardo Pedro Buera (AIR 46, 21/10/1976, folio 154, y Ministerio de Cultura y Educación, Resolución No 1546, 5/11/1976, folio 151). Pocos días después, Berenguer Carisomo rechazó las renuncias presentadas por varios directores de Departamento, entre ellas la de María del Carmen Carlé (AIR 46, 10/11/1976, folio 150).

Durante la última gestión de Caillet Bois al frente del Instituto Ravignani, fueron habituales las comunicaciones con el Rectorado de la UBA y con la FFyL, por cuestiones administrativas y/o académicas, como el pedido del delegado militar de la UBA, capitán de navío Edmundo Said, de elevar al Departamento de Personal el listado y las fotografías de todo el personal, docente y no docente, del Instituto (AIR 46, 1/5/1976 y 31/5/1976, folios 42 y 41), la solicitud de información por parte de la Secretaría de Asuntos Académicos acerca de las investigaciones proyectadas y en curso (AIR 98, 11/5/1976, folio 1), la comunicación del aumento de la cuota de descuento voluntario para ahorro (AIR 46, 28/7/1977, folio 5), de las nuevas disposiciones referentes a pedidos de licencias y/o solicitudes para viajes al exterior (AIR 46, 3/8/1976, 6/10/1976, 17/11/1976 y 19/11/1976, folios 141-146), y/o de la inscripción a los cursos organizados por la Dirección General de Personal de la UBA (AIR 46, 22/2/1977 y 6/6/1977, folios 80 y 37); así como la solicitud de enviar a la Facultad las copias legalizadas de los títulos y/o certificados de especialización o capacitación de todo el personal a su cargo (AIR 46, 12/5/1976, folio 217), las notas donde Berenguer Carisomo le solicitaba a Caillet Bois vigilar por motivos económicos las llamadas telefónicas de su dependencia (AIR 46, agosto de 1977, folio 3), cumplir con la centralización de todas las comunicaciones en la Oficina de Prensa de la Facultad (AIR 46, 10/12/1976 y 14/3/1977, folios 137 y 71 ), y hacer cumplir una serie de medidas relativas a la presentación del personal en su lugar de trabajo, la prohibición de ausentarse sin autorización y de permanencia de toda persona ajena a la dependencia sin justificación, de hacer llamadas telefónicas de índole personal, de usar radios y grabadores y de vender mercaderías (AIR 46, 14/2/1977, folio 85), y la obligación de los agentes de la Facultad de acreditar su identidad al ingreso mediante la credencial correspondiente (AIR 46, 21/4/1977, folio 60).

Caillet Bois también se encargó de gestionar tanto la reincorporación de su antiguo colaborador, Julio César González; ${ }^{13}$ como los fondos necesarios para retomar la publicación del Archivo de Rufino de Elizalde, en particular, los tomos V y VI que ya estaban corregidos y listos para su impresión en la imprenta del Congreso de la Nación, ante el delegado militar, capitán de corbeta Claisse, y el decano Battistessa, no sin destacar la subvención inicial que supo brindar el teniente general Juan Carlos Onganía y recordar que los últimos volúmenes fueron publicados "cuando la Facultad de Filosofía y Letras había caído en poder de Ortega Peña y Eduardo Luis Duhalde, que nada hicieron para continuar con la publicación” (AIR 107, 3/6/1976 y 12/8/1976, s/f). Caillet Bois fue asimismo designado para integrar la Comisión Honoraria encargada de organizar la conmemoración del octogésimo aniversario de la creación de la Facultad (AIR 46, 6/8/1976, folio 172). 
Bajo su nueva gestión no dejó de implementarse la usual política de canjes y donaciones. Por casos, se encuentran tanto la solicitud -en carácter de donación- de una serie de libros para la biblioteca del Instituto a la Editorial Universitaria de Buenos Aires (AIR 44, 13/10/1976, folio 187); como el pedido de préstamo de materiales cursado por la Academia Argentina de Letras, solicitando el primer tomo del Archivo histórico del Brigadier Juan F. Quiroga (AIR 44, 29/10/1976, folio 89).

Con fecha 15 de junio de 1977, se encuentra una nota con el personal del Instituto, donde se consignaban sus nombres y números de legajos: Julio C. González, Aída Selaroff, Olga García de D 'Agostino, Juan Severino López, Elena Rebok, Norma Asato, Paulino R. Vázquez y Adriana G. Cortijo. ${ }^{14}$

$\mathrm{Al}$ parecer, entonces también fueron muy habituales las presentaciones de informes de investigación como los de Asato sobre la "cuestión brasileña", centrados en la consulta de archivos y bibliotecas (AIR 98, 3/6/1977, folios 71-79; 19/7/1976, folios 65-69; 22/9/1976, folios 57-64; 22/12/1976, folios 42-49; 14/3/1977, folios 11-18; 3/8/1977, folios 80-81); los de López acerca del "problema de la frontera sobre el Chaco en la época de la Confederación Argentina”, donde se limitaba a especificar sus trabajos en curso y los que ya estaban listos para ser publicados (AIR 98, 28/12/1976, folio 82); los de Rebok centrados no sólo en la consulta de materiales de archivo, sino en su inventario, fichaje y traducción, así como en materiales sobre los cónsules argentinos en Alemania y en sus trabajos sobre los primeros pasos del socialismo y el anarquismo en la Argentina a través de fuentes documentales alemanas que pudo consultar durante su estancia de investigación allí, en particular, del Archivo Político del Ministerio de Relaciones Exteriores de Bonn, en Alemania Federal, y el periódico Vorwärts (AIR 98, 1/10/1976, folios 83-85; 1/11/1976, folios 86-90; 1/12/1976, folios 91-113; 31/12/1976, folios 114-122; 1/3/1977, folios 123-124; 1/4/1977, folios 125-134; 1/5/1977, folios 135-147; 1/6/1977, folios 148-161; y 1/7/1977, folios 162-176); y los de García de D 'Agostino sobre los materiales existentes en el Instituto Ravignani tanto del Archivo de Viena sobre la correspondencia "De Cónsules Austríacos al Canciller del Estado (1816-1818)" y los fondos francesesAsuntos Exteriores de Francia referidos a las islas Malvinas, así como materiales sobre legaciones extranjeras en el Archivo de Relaciones Exteriores y Culto, en particular, los correspondientes a Perú, Bolivia y Chile desde 1874 hasta 1879, y un trabajo sobre las relaciones internacionales durante la segunda mitad del siglo XVIII (1764-1780) (AIR 98, 1/10/1976, folios 177-179; 3/11/1976, folios 180-183; 3/12/1976, folios 184-189; 30/12/1976, folios 190-192; 2/3/1977, folios 193-198; 30/4/1977, folios 199-203; 1/6/1977, folios 204-211; y 4/7/1977, folios 212-221). En casi todos ellos aparece consignada la aprobación del director, escrita de su puño y letra.

En la Memoria institucional de 1977 se especificó que el personal de investigación del Instituto estaba integrado por las profesoras García de D'Agostino, Rebok y Asato; que el personal no docente estaba integrado por el profesor López - que cumplía tareas de investigación-, Selaroff como bibliotecaria y Vázquez; y se aclaraba que la profesora Liliana E. Darriba pertenecía al personal del Instituto de España, pero que prestaba transitoriamente servicios en el Instituto a partir del 6 de septiembre.

Por otra parte, se daba cuenta de las investigaciones realizadas hasta el 31 de agosto, fecha en la que asumió sus funciones, tras el fallecimiento de Caillet Bois el 16 de julio, la nueva directora, Daisy Rípodas Ardanaz. ${ }^{15}$ Se hacía alusión a una investigación en equipo sobre el tema "La conquista del desierto. De Rosas a Roca (1829-1879)", advirtiendo que estaba en su etapa heurística y distribuida del siguiente modo: "La época de Rosas (1829-1852)" a cargo de Asato, "De Urquiza a Sarmiento (1852-1872)" por López, y "La presidencia de Avellaneda (1874-1880)” por García de D'Agostino y Rebok. Se aclaraba que los investigadores mencionados habían iniciado asimismo el fichado de la documentación colonial conservada en el Instituto. También se especificaban las investigaciones individuales: las investigaciones concluidas de García de D'Agostino sobre "Aspectos de las relaciones de la República Argentina con Bolivia, Chile y Perú (1874-1879)" y de López sobre "Las fronteras interiores y los indios en la época de la Confederación Argentina (1852-1861)"; y la investigación en curso de Rebok sobre "La política alemana (aspectos económicos, políticos y culturales) en la República Argentina durante los siglos XIX y XX”. ${ }^{16}$ 
De hecho, en el AIR se encuentran los "Informes del personal de investigación al 31/8/1977", donde Asato, García de D'Agostino, López y Rebok dieron cuenta de sus temas de investigación y la documentación consultada: la política brasileña con relación a los intereses económicos y a la política de frontera (siglos XIX-XX) (Asato), los fondos franceses del Instituto Ravignani integrados por correspondencia política de Asuntos Exteriores de Francia, es decir, los informes de los ministros franceses en Londres en tanto aproximación a la posición de la corona británica sobre sus posesiones en los mares, en particular, acerca de las islas Malvinas (García de D 'Agostino), sus trabajos sobre las fronteras interiores a mediados del siglo XIX (López), y las relaciones argentino-germanas 1866-1936 (Rebok). ${ }^{17}$

Respecto del Boletín institucional, sabemos que hasta 1970 se publicaron con regularidad y una frecuencia anual los números de la llamada segunda serie (hasta el No22-23) (Guber y Rodríguez, 2011, pp. 75-76). El $\mathrm{N}^{\circ}$ 24-25, correspondiente al período 1970-1971, fue impreso finalmente en 1973, y para el $\mathrm{N}^{\circ} 26$, cuyos originales datan de 1978, hubo que esperar para su publicación hasta el año $1980 .{ }^{18}$ En este último número es posible observar el homenaje realizado tanto a Caillet Bois como a González tras sus fallecimientos en 1977 y 1978, en forma respectiva. Si bien, a modo de homenaje, se decidió publicar trabajos de sus discípulos personales, de investigadores del Instituto y de colaboradores del Boletín, también se recordó su paso por las cátedras de Historia Argentina y de Historia de América independiente, la "exitosa" dirección del Instituto por parte del primero así como su título de doctor honoris causa por la UBA, la "entrañable amistad” que los unía, y cómo González supo secundar y hasta reemplazar a Caillet Bois en las tareas del Instituto así como estar al frente de la Biblioteca Central de la Facultad (D.R.A., 1980, pp. 9-11).

\section{A MODO DE CONCLUSIÓN}

Las universidades públicas, en general, y la UBA y sus dependencias, en particular -como el caso de la FFyL-estuvieron inmersas en el contexto de fuerte represión e inestabilidad político-institucional que transitó la Argentina durante el gobierno de Isabel Perón y los primeros años de la última dictadura. En buena medida, coincidimos con la idea de una "transición a la dictadura" tal como sostiene Sergio Friedemann (2016) en alusión al carácter represivo que impregnó a la UBA desde la intervención de septiembre de 1974 -la misión Ivanissevich- en la medida que "allanó el camino a la sistematización de la represión ilegal” (pp. 12-13), recordando al mismo tiempo la existencia de una represión previa y que la ejercida a partir del golpe lo hizo sobre un ámbito universitario ya diezmado (Besoky, 2017).

En el presente artículo nos hemos interrogado acerca de su impacto en un ámbito mucho más restringido, como el del Instituto de Historia Argentina y Americana. Nuevamente debemos precisar que al inicio de nuestra indagación el Instituto no existía como tal sino que momentáneamente había constituido el Centro de Estudios de Historia Argentina y Latinoamericana, dependiente del también entonces recientemente creado Instituto de Estudios Argentinos y Latinoamericanos.

Como hemos visto, el Instituto fue restituido hacia fines de 1974 cuando se dispuso la reorganización académica e institucional de la Facultad. Entonces conservó el nombre de Diego Luis Molinari pero se antepuso el título de "Dr.". Al parecer, tras unos meses de acefalía y la gestión informal de Ocón, en agosto de 1975 no sólo el Instituto se convirtió en una unidad de investigación departamental, sino que fue nombrado oficialmente como su director Ibarguren. Tras la renuncia de Ibarguren, en mayo de 1976 se materializó el regreso de su antiguo director, Caillet Bois, secundado luego por González, así como el renombramiento del Instituto como "Dr. Emilio Ravignani". Justamente, nuestra indagación concluye con su muerte a mediados de julio del año siguiente.

En cuanto a la adscripción política e historiográfica de Ibarguren y Caillet Bois, si bien a nivel político ambos pueden ser de algún modo enrolados en el amplio abanico de las derechas argentinas, en lo que respecta a su filiación historiográfica el primero respondía al revisionismo mientras que el segundo a la NEH. Por 
otra parte, la apertura hacia el revisionismo puede verse en algunas de las actividades organizadas durante las gestiones de Ocón e Ibarguren al frente de la Sección Historia y del Instituto, respectivamente.

A diferencia de la inestabilidad que se advierte en sus cuadros directivos, en parte de su funcionamiento y en el mismo cambio de nomenclatura, se puede observar cierta estabilidad en lo que respecta a su personal administrativo y técnico. Esa estabilidad no se evidencia en todo su elenco, aunque esta aseveración merece una serie de precisiones. De hecho, el plantel de investigadores del Instituto se había visto fuertemente renovado durante las gestiones anteriores de la izquierda peronista, que en buena medida habían operado de manera sumatoria, es decir, apelando más a los nuevos nombramientos que a las cesantías. Con los cambios organizativos y de gestión apuntados, gran parte de aquellos investigadores quedó fuera del Instituto, y hemos visto cómo Caillet Bois, en particular, procuró rodearse de sus antiguos colaboradores.

Algo similar sucedió con sus investigaciones y proyectos de investigación en la medida que se observa cierta ruptura respecto de las aperturas temáticas, problemáticas, de enfoques y de marcos conceptuales y metodológicos que se habían propuesto en ese momento de renovación que supuso la coyuntura de 1973-1974. Nuevamente, bajo la gestión del último se puede observar cómo se retomaron en parte viejos temas, problemáticas y enfoques.

En todo el período, en cambio, se observa la preocupación por proseguir con la publicación de las series documentales del Instituto, en especial, con el Archivo del Doctor Rufino de Elizalde; así como cierta distensión paulatina en las relaciones entre el Instituto y el Departamento de Ciencias Históricas o luego de Historia.

\section{Publicaciones PeRiódicas}

Boletín del Instituto de Historia Argentina y Americana "Dr. Emilio Ravignani”, XIV-XV(24-25). Segunda serie. (1980). Boletín del Instituto de Historia Argentina y Americana "Dr. Emilio Ravignani”, XVI(26). Segunda serie.

\section{FuenTES}

Archivo del Instituto Ravignani (AIR), Cajas N 43, 44, 46, 98, 101 y 107.

\section{REFerenCias BIBLIOgRÁFICAS}

Besoky, J. L. (2017). La gestión del ministro Ivanissevich y la derecha peronista: los 100 días de Ottalagano. Folia, 29, 145-174. Recuperado de http://www.scielo.org.ar/scielo.php?script=sci_arttext\&pid=S0325-8238201700020 0007\&lng=es\&nrm=iso\&tlng=es

Buchbinder, P. (1997). Historia de la Facultad de Filosofiay Letras, Universidad de Buenos Aires. Buenos Aires: Editorial Universitaria de Buenos Aires

Buchbinder, P. (2007). Emilio Ravignani: la Historia, la Nación y las Provincias. En F. Devoto (Estudio preliminar y comp.) La historiografia argentina en el siglo XX (pp.109-151). Buenos Aires: Editores de América Latina.

Devoto, F. (2006). Introducción. En Devoto (Estudio preliminar y comp.) La historiografía argentina en el siglo XX (pp. 5-35). Buenos Aires: Editores de América Latina.

Devoto, F. y Pagano N. (2009). Historia de la historiografía argentina. Buenos Aires: Sudamericana.

D.R.A. (1980). Advertencia. Boletin del Instituto de Historia Argentina y Americana "Dr. Emilio Ravignani", XVI(26). Segunda serie. 
Friedemann, S. (2016). Transición a la dictadura durante el gobierno de Isabel Perón. El ocaso de la Universidad Nacional y Popular de Buenos Aires. Revista de la carrera de Sociologia, 6(6), 3-36. Recuperado de https://publi caciones.sociales.uba.ar/index.php/entramadosyperspectivas/article/view/1573/2163

García Moral, M. E. (2010). El revisionismo en los 80 y 90: ¿el anquilosamiento o la convalecencia de una historia militante? En F. Devoto (Dir.), Historiadores, ensayistas y gran público. La historiografía argentina en los últimos veinte años (1990-2010) (pp. 79-96). Buenos Aires: Biblos.

Guber, R., y Rodríguez, M. (2011). Vitrinas del mundo académico: Las revistas de la Facultad de Filosofía y Letras de la Universidad de Buenos Aires entre 1946-1966. Historiografias, 2, 66-84.

Mallimaci, F., y Giorgi, G. (2007). Nacionalismos y Catolicismos en la Facultad de Filosofía y Letras de la Universidad de Buenos Aires. En Acta Académica, VII Jornadas de Sociología (pp. 1-13). Facultad de Ciencias Sociales Universidad de Buenos Aires, Buenos Aires. Resumen recuperado de http://cdsa.aacademica.org/000-106/32 2.pdf

Pagano, N. y Galante, M. (1993). La Nueva Escuela Histórica: una aproximación institucional del Centenario a la década del 40. En F. Devoto (Comp.), La historiografia argentina en el siglo XX (pp. 45-78). (tomo 1). Buenos Aires: Centro Editor de América Latina.

Ramallo, J. M. (1984). La Revista del Instituto Rosas (1939-1961). Noticia, indice y textos. Buenos Aires: Fundación Nuestra Historia.

Seia, G. A. (s.f.). Rectores de la Universidad de Buenos Aires entre 1974 y 1983: curricula vitae, discursos y comunicados de prensa", pp. 1-12. Recuperado de http://www.uba.ar/archivos_internacionales/image/RECTORES\%20DE\%20LA\%20UNIVERSIDAD\%2 0DE\%20BUENOS\%20AIRES\%20ENRRE\%201973\%20Y\%20\%201983.pdf

Seia, G. A. (2015). La formación de los profesores de Historia en la Universidad de Buenos Aires entre 1973 y 1983. Revista de Historia, 16, 78-101. Recuperado de http://revele.uncoma.edu.ar/htdoc/revele/index.php/historia/ index

\section{Notas}

1 Nos referimos a la ponencia "De Ravignani a Molinari: el paso de la izquierda peronista por el Instituto de Historia Argentina y Americana (1973-1974)", presentada en las II Jornadas Internacionales de historia del movimiento obrero y la izquierda, organizadas por el Departamento de Historia y el Instituto de Historia Argentina y Americana "Dr. Emilio Ravignani” de la FFyL de la UBA y celebradas en Buenos Aires entre el 3 y el 5 de octubre de 2018.

2 Durante el período que nos ocupa ellos fueron: Ricardo Pedro Bruera (marzo de 1976 - abril y mayo de 1977), Harguindeguy (mayo - junio de 1977) y Juan José Catalán (junio de 1977 - agosto de 1978). Seia, s/f., pp. 6-7.

3 Resolución $\mathrm{N}^{\circ} 93$ (bis), 28/12/1974. En octubre de 1975, Bórmida pidió autorización para ausentarse con el objetivo de realizar una investigación etnográfica en el Chaco Boreal Boliviano. En su lugar fueron designados al frente del Departamento de Ciencias Históricas y del Instituto de Ciencias Antropológicas Horacio Difrieri y Amalia Sanguinetti de Bórmida, respectivamente. AIR 46, 16/10/1975, folio 279.

4 Dependían del mencionado Instituto los siguientes Centros de Estudios: de la Cultura Popular, de la Realidad Educativa, Socio-Económicos, Sociales de la Comunicación, Sociales del Trabajo, Sociales de la Salud y el de Historia Argentina y Latinoamericana.

5 Ocón había presidido en sus inicios el Centro de Estudios de Historia Argentina (CEHA) -la actual Fundación Nuestra Historia-, creado por Jorge M. Ramallo y Enrique Guerrero Balfagón en 1963 en tanto ramificación del Instituto de Investigaciones Históricas "Juan Manuel de Rosas", de corte conservador y católico (García Moral, 2010, pp. 80 y 82 ; Ramallo, 1984, p.11).

6 Como el de la profesora marplatense Nora Ester Cisilino, que tuvo una respuesta positiva, así como el de Raúl García Heras. AIR 43, 19/7/1975, 25/7/1975 y 30/6/1975, folios 47, 54 y 226.

7 AIR 43, 8/7/1975 y 25/7/1975, folios 238 y 243. En la respuesta afirmativa del pedido se especifica la condición de Ocón como director del Instituto Molinari.

8 AIR 43, 25/7/1975, folio 274. Previamente, Vázquez había sido designado para inventariar los bienes muebles existentes en el Instituto. AIR 43, 5/6/1975, folio 122. La preocupación por el inventario de bienes era habitual. Abelenda había sobreseído provisionalmente por falta de pruebas el sumario administrativo dispuesto en marzo de 1974 en virtud de la desaparición de una máquina de escribir del Instituto. Resolución Nº 336, 14/5/1975. 
9 AIR 43, 10/4/1975, folio 334. En la respuesta que buscaba dar satisfacción a su pedido se indicaba que Ocón era el director de la Sección Historia del Instituto de Historia Argentina y Americana “Dr. Diego Luis Molinari”. AIR 43, 4/5/1975, folio 335 y $6 / 5 / 1975$, folio 333 .

10 AIR 46, Resolución No 1097, 27/8/1975, folios 296-298. Durante su gestión, también se respondió afirmativamente el pedido de publicaciones institucionales remitido por la Presidencia de la Nación, Casa Militar- Museo de la Casa de Gobierno. AIR 43, 11/12/1975 y 18/12/1975, folio 297.

11 Se hacía referencia a la necesidad de reordenamiento de los institutos y centros de estudio y de investigación de la Facultad en lo que refería a su existencia, denominación y dependencia -directa del decanato-. AIR 46, Resolución No 232, 12/5/1976, folios 206-207.

12 AIR 46, Resolución No 243, 12/5/1976, folio 215. En junio se le asignó una renta de profesor titular por el desempeño de dichas funciones, que se le quitó en febrero del año siguiente. AIR 46, Resolución N 33, 14/2/1976, folio 86.

13 En septiembre se aceptó el pedido de Caillet Bois de designación de Julio César González. Se trató de una designación interina como investigador asimilado a la categoría de profesor titular con dedicación simple del IHAyAER, por el período comprendido entre el $1^{\circ}$ de septiembre de 1976 y el 31 de marzo de 1977 . AIR 46, 13/9/1976, folio 159.

14 AIR 44, 15/6/1977, folio 295. Mientras que los nombres de González y Cortijo aparecen tachados, se agrega el de Liliana Darriba.

15 AIR 46, Resolución No 984, 22/8/1977, folio 2. Ese mismo día, el decano Berenguer Carisomo designó a Julio C. González miembro honorario del IHAyAER. AIR 46, Resolución No 985, 22/8/1977, folio 1.

16 AIR 101, “1977. Memoria del Instituto de Historia Argentina y Americana 'Dr. Emilio Ravignani'”, 13/12/1977, folios 128-131.

17 AIR 98, Asato, Norma, García, Olga, López, Juan Severino, Rebok, Elena, "Informes del personal de investigación al 31 de agosto de 1977", folios 50-55.

18 Se ha consultado la segunda serie del Boletín del Instituto de Historia Argentina y Americana "Dr. Emilio Ravignani”, en particular los $\mathrm{N}^{\circ} 24-25$ y 26, de 1973 y 1980, respectivamente. 